\title{
Research Paper: \\ The Effect of Omega-lycotoxin on the Cognitive Impairment Induced by Kainic Acid in Rats
}

\author{
Ali Hosseini-Sharifabad ${ }^{1}$ (D), Mohammad Reza Mofid² (D, Majid Moradmand ${ }^{3}$ (D), Mohammad Keimasi ${ }^{1^{*}}$ (D)
}

1. Department of Pharmacology and Toxicology, Isfahan Pharmaceutical Sciences Research Center, School of Pharmacy and Pharmaceutical Sciences, Isfahan University of Medical Sciences, Isfahan, Iran.

2. Department of Clinical Biochemistry, Bioinformatics Research Center, School of Pharmacy and Pharmaceutical Sciences, Isfahan University of Medical Sciences, Isfahan, Iran.

3. Department of Animal and Plant Biology, Faculty of Biological Sciences and Technology, University of Isfahan, Isfahan, Iran.

\begin{tabular}{|l|l|l|l}
\hline $\begin{array}{l}\text { Use yourdevice tosan } \\
\text { and read the artice online }\end{array}$ & $\begin{array}{l}\text { How to cite this paper Hosseini-Sharifabad A, Mofid MR, Moradmand M, Keimasi M. The Effect of Omega-lycotoxin } \\
\text { on the Cognitive Impairment Induced by Kainic Acid in Rats. Iranian Journal of Toxicology. 2021; 15(1):49-56. http://dx.doi. } \\
\text { org/10.32598/ijt.15.1.740.1 }\end{array}$ \\
dol http://dx.doi.org/10.32598/ijt.15.1.740.1
\end{tabular}

(c) (i) (3)

Article info:

Received: 07 Aug 2020

Accepted: 20 Sep 2020

Online Published: 01 Jan 2021

* Corresponding author:

Mohammad Keimasi, MSc.

Address: Department of Pharmacology and Toxicology, Isfahan Pharmaceutical Sciences Research Center, School of Pharmacy and Pharmaceutical Sciences, Isfahan University of Medical Sciences, Isfahan, Iran.

E-mail: keimasimohammed@gmail.com

\section{A B S T R A C T}

Background: Excitotoxicity is a common pathological process in neurodegenerative diseases associated with overactivity of N-methyl-D-aspartate (NMDA) and P/Q type voltage-gated calcium (Cav2.1) channels. Omega-lycotoxin-Gsp2671g is a therapeutic tool to modulate overactive Cav2.1 (P/Q type) channels. Omega-lycotoxin binds to Cav2.1 channels with high affinity and selectivity. This study aimed to investigate the effects of Omega-lycotoxin on the cognitive impairment induced by kainic acid in rats.

Methods: The effect of pre-treatment and post-treatment trials of intra-hippocampal Cornu Ammonis-3 administration of omega-lycotoxin $(0.5,1$ or $2 \mu \mathrm{g})$ was studied on the cognitive impairment induced by kainic acid in rats. The rats' learning and memory were assessed by the passive avoidance and a single-day testing version of the Morris water maze method.

Results: Omega-lycotoxin caused a significant increase in the latency of the passive avoidance test and the duration of their presence in the target area of the Morris water maze test compared to the groups treated with kainic acid $(\mathrm{P}<0.0001)$. There were statistically significant differences for the effects of various doses of omega-lycotoxin. The post-treatment groups showed a greater improvement than those in the pretreatment groups.

Conclusion: The findings demonstrated that a single dose of omega-lycotoxin can prevent or revert the memory impairment caused by kainic acid in rats.

Keywords: Calcium channel blockers, Kainic acid, Memory \& avoidance tests, Calcium channel Cav2.1 (P/Q type)

\section{Introduction}

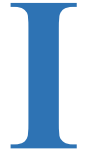

$\mathrm{n}$ recent years, neurodegenerative conditions, such as Alzheimer's, Parkinson's and Huntington's diseases have spread worldwide, and the prevalence is rapidly increasing [1]. These diseases are progressive and disrupt the daily activities of many patients with such side effects as amnesia, paralysis, motor disorders, and eventually death $[1,2]$. The causes of these diseases are still unclear but they may stem from genetic and epigenetic factors, diet, and lifestyle $[3,4]$. 
In the central nervous system of mammals, P/Q-type of voltage-gated calcium channels ( $\mathrm{Cav} 2.1$ ) control calciumdependent processes, such as synaptic plasticity and neurotransmitters release $[5,6]$. These channels are located at the presynaptic terminals and initiate the fusion of presynaptic vesicles by the influx of calcium ions into nerve endings $[4,5]$. The neurotransmitters that are released from the pre-synaptic vesicles enter the synaptic clefts $[7,8]$.

Ionotropic and metabotropic N-methyl-D-aspartate (NMDA) receptors are located at post-synaptic terminals. These receptors are classified into three categories: A. N-methyl-D-aspartate (NMDA); B. Alphaamino-3-hydroxy-5-methyl-4-isoxazole propionic acid (AMPA); and C. Kainic acid receptors [9, 10]. Through these receptors, calcium, potassium and/or sodium ions enter neurons. These receptors play an important role in learning and memory $[11,12]$. They support dendrites and synapses in the brain and their excitotoxicity effects can result in overactivity of the ion channels, leading to destructive effects on the brain cells [13]. Also, calcium ions play an essential role in the release of glutamate. Many studies show that excessive activity of glutamate receptors has a pivotal role in the development and progression of neurological disorders and neuronal toxicity, known as excitotoxicity [11, 14-16]. These studies suggest that excessive stimulation of glutamate receptors following ischemic stroke, cerebral hemorrhage, and epilepsy, play a key role in the development of such pathologies as amnesia $[9,17,18]$.

In many studies, kainic acid has been used as a systemic or intrahippocampal administration to cause excitotoxicity, which is very similar to neuronal toxicity in epilepsy, Alzheimer's, and other neurodegenerative diseases [1921]. Therefore, one of the main manifestations of this toxicity is amnesia. This overstimulation may also be due to increased neurotransmitter release from the nerve endings [10, 19, 20, 22]. The excessive stimulation of glutamate receptors causes large amounts of calcium to enter the neurons, which can activate inflammatory enzymes such as cyclooxygenase or nitric oxide synthase and tumor necrosis factor, and finally can lead to neuronal inflammation and damage $[10,11]$.

On the other hand, over-saturation of mitochondrial calcium generates various types of free radical species, activation of the intrinsic apoptotic pathway, and P38 factor which causes neuronal necrosis. These events are involved in the induction of neuronal death rather than their plasticity [10]. Kainic acid can cause the release of large amounts of neurotransmitters in pre-synaptic spac- es by overactivity of CaV2.1 channels. The increased neurotransmitters release leads to excitotoxicity $[19,20]$.

At present, effective and safe drugs are scarce for the prevention or treatment of diseases like Alzheimer's and epilepsy. The existing drugs, such as memantine and gabapentin have low-affinity for their target receptors and may cause adverse effects. Currently, there is not an effective drug for preventing or treating neuronal degeneration. Also, the existing drugs including NMDA inhibitors (e.g. memantine) do not have a very high affinity for the receptor and are considered as non-selective antagonists with adverse effects. Gabapentin is used to block the voltagedependent calcium channels. These drugs can be used in moderate but not severe conditions $[1,2,9,23,24]$.

Using natural compounds to prevent the formation or progression of neurodegenerative diseases by modifying the underlying mechanisms or repairing the neuronal damage are viewed as promising therapeutic approaches. Research has shown that omega-lycotoxin, a toxin from wolf spider, can modulate CaV2.1 channels in rat cerebellar Purkinje cells and hippocampal CA1-CA3 neurons $[25,26]$. However, there are no studies on its behavioral assessments reflecting memory retention and learning. Omega-lycotoxin can play a modulating role in cases of excessive stimulation of pre-synaptic calcium channels induced by kainic acid. This study aimed at investigating the effect of pre- and post-treatment of the hippocampal neurons with omega-lycotoxin in rats and the assessment of the resultant cognitive impairment caused by exposure to kainic acid.

\section{Materials and Methods}

Chemicals \& reagents: Omega-lycotoxin-Gsp 2671g was supplied by MyBioSource Company (San Diego, USA). Kainic acid, ketamine, and xylazine were purchased from Sigma Aldrich Company (Munich, Germany).

Animals: A total of 54 adult male Wistar rats, weighing 230-250 g were obtained from the animal house of the Faculty of Pharmacy, Isfahan University of Medical Sciences. All rats were kept in standard cages under controlled environmental conditions of humidity ( $\sim 60 \%)$, temperature $\left(\sim 25^{\circ} \mathrm{C}\right), 12 \mathrm{hr}$. of light-dark cycle, and free access to food and water ad libitum. The experimental protocol observed all of the internationally accepted guidelines concerning the handling and care of experimental animals.

Experimental design: The study was conducted on adult male rats, randomly divided into nine groups of six rats each. The PBS buffer consisted of $137 \mathrm{mM} \mathrm{NaCl}$, 
$3 \mathrm{mM} \mathrm{KCl}, 10 \mathrm{mM} \mathrm{Na} 2 \mathrm{PO} 4,2 \mathrm{mM}$, KH2PO4, at $\mathrm{pH} 7.4$ $[27,28]$. This buffer was used as a vehicle for omegalycotoxin, while normal saline was used as a vehicle for kainic acid. Each experiment was performed in the following order:

- Control group: Received a dose of $2 \mu 1$ normal saline in the hippocampus and was injected $2 \mu 1$ PBS intrahippocampal 20 minutes later.

-Kainic acid pre-treated group: Received a single dose of kainic acid $(2 \mu \mathrm{g}, 1 \mathrm{mg} / \mathrm{ml})$ followed 20 minutes later by an injection of $2 \mu \mathrm{PBS}$ in the hippocampus [29].

- Kainic acid post-treated group: Received a dose of $2 \mu 1$ PBS followed by a single dose of kainic acid $(2 \mu \mathrm{l}, 1$ $\mathrm{mg} / \mathrm{ml}$ ) in the hippocampus 20 minutes later.

- Omega-lycotoxin pre-treated groups: The three groups of animals received either $0.5,1$, or $2 \mu \mathrm{g}$ doses of omega-lycotoxin followed by a single dose of kainic acid $(2 \mu \mathrm{g})$ in the hippocampus 20 minutes later.

- Omega-lycotoxin post-treated groups: The three rat groups received a single dose of Kainic acid $(2 \mu \mathrm{g})$ followed by an injection of either $0.5,1$, or $2 \mu \mathrm{g}$ of omega-lycotoxin in the hippocampus 20 minutes later.

Surgery: On the day of surgery, each rat was weighed and anesthetized with i.p. injection of a mixture of Ketamine and Xylazine (100 and $2.5 \mathrm{mg} / \mathrm{kg}$, respectively). Adequate lidocaine and epinephrine were injected subcutaneously at the site of surgery to induce local anesthesia and prevent bleeding.

Micro-injection procedure: The agents were administered into the CA3 zone of the right hemisphere by using a $5 \mu \mathrm{l}$ Hamilton syringe. The site of injections was identified on the skull of each rat as follows: AP: $-3.3 \mathrm{~mm}$ from bregma; ML: $-3 \mathrm{~mm}$ from the midline; DV: 3.5 $\mathrm{mm}$ from the skull surface based on the Paxinos and Watson's Atlas of the rat brain [30]. The needle was inserted into the marked area and the agents were injected slowly over $6 \mathrm{~min}$. Two minutes after injection, the needle was slowly removed to avoid backflow of the fluid.

Behavioral assessments: The rats' behavioral assessments were performed seven days after the surgery. The behavioral assessments included "passive avoidance" and "Morris water maze" tests. These tests evaluated the passive avoidance, spatial memory, and learning, respectively, in each animal.
Passive Avoidance Test: The passive avoidance test is classically designed to evaluate the memory components of the learning process and the emotional memory in animal models for neurological disorders. Although searching in a dark place is an inherent need for rodents, the animal learns that it will be shocked if entering the dark compartment during the training phase.

Passive avoidance learning was assessed during the animals crossing through a shuttle box. It had two compartments with an up-down sliding door on top of each compartment. One of these compartments was a light chamber with white walls and the other was a dark chamber with black walls. Another sliding door was located in the middle between the light and dark sections. Stainless steel shocker rods covered the floor of the dark chamber, which were connected to an electroshock device by a live wire. The intensity, frequency and duration of the current were fully adjustable.

The evaluation of the passive avoidance behavior was performed on two consecutive days. The first training day had two sections: habituation and experimental sessions.

- Habituation: The animals were kept in the laboratory for at least 30 minutes to get accustomed to the new environment before starting the experiment.

- Experiment: The gate was slid up and the animal was allowed to enter the dark chamber from the initial light chamber. The initial latency for the animal before entering the dark chamber was recorded.

The criterion for the animals to enter the dark chamber was for the hind legs to cross into the dark compartment. After $10 \mathrm{sec}$, the animal was returned to its cage. Animals with a latency of $100 \mathrm{sec}$ or longer were excluded from the study. After 30 minutes, the animals were returned to the light chamber one at a time and the sliding gate opened after $5 \mathrm{sec}$. When each animal entered the dark chamber, the gate was slid down and the rat was shocked $(1 \mathrm{~mA}, 50 \mathrm{~Hz}, 3 \mathrm{sec})$ through the floor bars. Two minutes later, each rat was taken back to the light compartment again and the process was repeated. If the rat did not enter the dark section, the test was over for that animal. Otherwise, the same process was repeated until they did not enter the dark chamber. One day after the training, the rats were returned to the light chamber separately and the latency to re-enter the dark chamber was recorded again as a means of evaluating their memory of the procedure they went through the day before [31]. 
Morris Water Maze Test: The Morris water maze test is a procedure to evaluate spatial memory and learning in rodents. The maze used in this study was a circular metal tank with black walls, and was filled with water to a height of $20-30 \mathrm{~cm}$. A plexiglass platform was placed $1-2 \mathrm{~cm}$ below the center of the target area, which was invisible to the animal being tested. The platform provided an opportunity for the animal to take a rest on it while swimming in the water tank. The walls around the tank had signs and symbols marked on them so the animals could use them to find the platform. The test included three phases of habituation, training and swimming.

A day before the training began, rats were habituated to the tank by allowing them to swim for $1 \mathrm{~min}$ without a platform. The one-day training included eight trials with four varying start points. Every rat was randomly entered into the water twice in each zone of the tank and allowed to swim and discover the underwater platform located in the southwest zone. After reaching the platform, the rats were let to stay there for 20 second and then were returned to the holding box to rest for $30 \mathrm{sec}$ before starting the next trial. The animals were then placed in the pool and they needed to learn the signs and other visual cues outside the maze to remember the location of a hidden platform below the water surface.

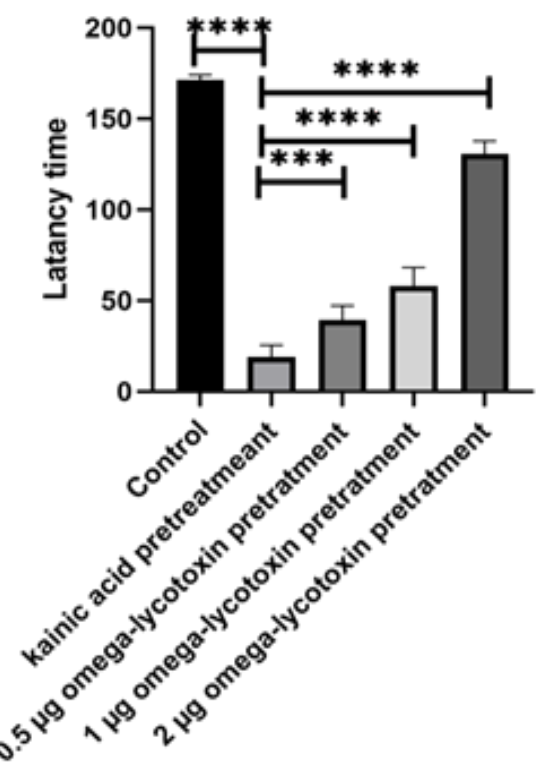

Groups

Figure 1. The passive avoidance test

The effect of pre-treatment groups of different doses of omega-lycotoxin on latency in the passive avoidance test. The data are shown as Means \pm SEM of 6 rats per group;

* $\mathrm{P}<0.05$; ${ }^{* *} \mathrm{P}<0.01$; *** $\mathrm{P}<0.001$; ${ }^{* * * *} \mathrm{P}<0.0001$.
After the initial training phase, the rats were returned to their home boxes for one day. In the probational part of the test, the hidden platform was removed and the rats were placed in the water from the north end and allowed to swim for one minute. Then the platform was set higher than the water surface and placed in the southeast area [32]. In this test, the time spent in the target zone was recorded and this factor was the criterion for the evaluation of spatial memory and learning.

Data Analysis: Statistical analysis was performed, using SPSS software, version 23. Primarily, the data were normalized by the Kolmogorov Smirnov test. Multiple comparisons were made of the data collected from the passive avoidance and the Morris water maze tests among the groups by one-way Analysis of Variance (ANOVA) and Tukey's Post Hoc tests. All data were shown as the means \pm standard errors of the means. For statistical analysis, $\mathrm{P}<0.05$ was considered a significant difference.

\section{Results}

Effect of Omega-lycotoxin vs Passive Avoidance Test: As shown in Figure 1, the administration of a single dose of kainic acid $(2 \mu \mathrm{g})$ into the hippocampus significantly decreased the latency time in the pre-treatment group

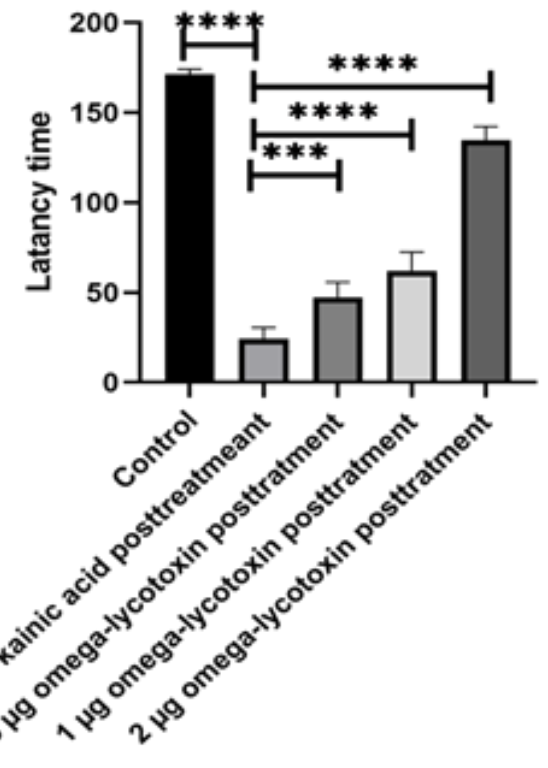

\section{Groups}

Figure 2. The passive avoidance test

The effect of post-treatment groups of different doses of omega-lycotoxin on latency in the passive avoidance test. The data are shown as Means \pm SEM of 6 rats per group;

${ }^{*} \mathrm{P}<0.05$; ** $\mathrm{P}<0.01$; *** $\mathrm{P}<0.001 ;$ **** $\mathrm{P}<0.0001$ 

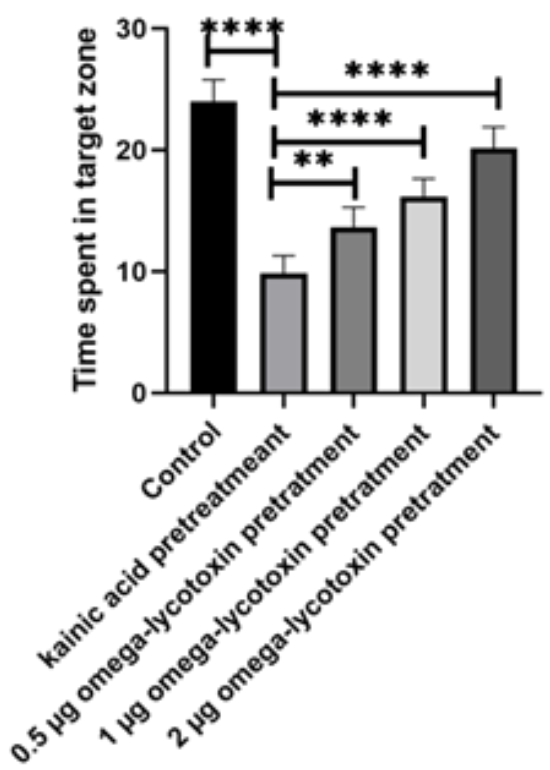

Groups

Figure 3. The Morris water maze test

The effect of pre-treatment groups of different doses of omega-lycotoxin on time spent in the target quadrant in the Morris water maze test. The data are shown as Means \pm SEM of 6 rats per group;

${ }^{*} \mathrm{P}<0.05$; ${ }^{* *} \mathrm{P}<0.01 ;{ }^{* * *} \mathrm{P}<0.001$; **** $\mathrm{P}<0.0001$.

compared to that noted for the control group $(\mathrm{P}<0.0001)$. All doses of omega-lycotoxin $(0.5-2 \mu \mathrm{g})$ significantly increased the latency compared to that of the kainic acid pretreatment group $(\mathrm{P}<0.001)$. Injection of the same doses of omega-lycotoxin increased the latency time for the passive avoidance test in the post-treatment group compared to that for the kainic acid-treated group. This agent at a dose of $2 \mu \mathrm{g}$ brought the latency time to near normal level $(\mathrm{P}<0.001)$ (Figure 2$)$.

Effect of Omega-lycotoxin on the Morris Water Maze Test: As shown in Figure 3, the injection of a single dose of kainic acid $(2 \mu \mathrm{g} ; 1 \mathrm{mg} / \mathrm{ml})$ in the hippocampus decreased the time spent in the target quadrant of the maze in the pre-treatment group as compared to that of the control group $(\mathrm{P}<0.0001)$. All doses of kainic acid $(0.5-2 \mu \mathrm{g})$ markedly increased the time spent in the target quadrant for the post-test group compared to that for this agent in the pretreatment group $(\mathrm{P}<0.0001)$. As shown in Figure 4, the administration of omega-lycotoxin (0.5$2 \mu \mathrm{g}$ ) increased the time spent in the target quadrant in the post-treatment group compared to that for the kainic acid in the post-treatment group $(\mathrm{P}<0.05)$ to an almost normal level $(\mathrm{P}<0.001)$.

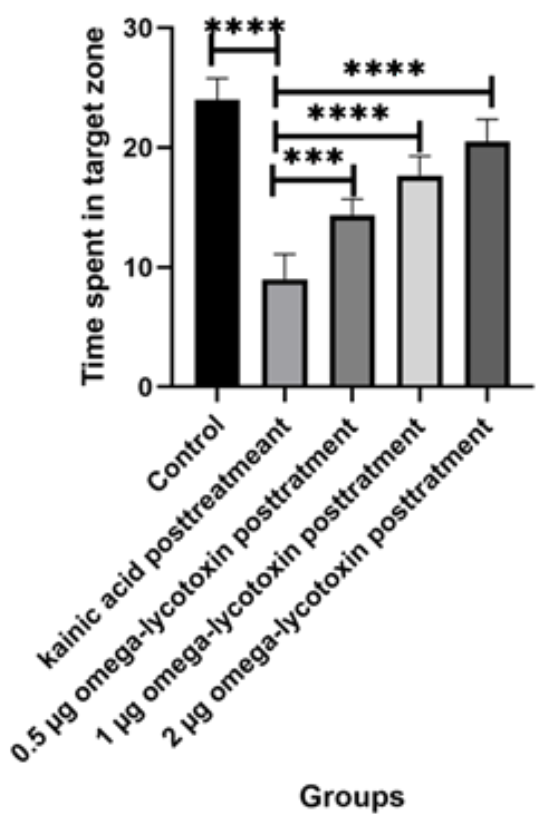

Figure 4. The Morris water maze test

The effect of post-treatment groups of different doses of omega-lycotoxin on time spent in the target quadrant in the Morris water maze test. The data are shown as Means \pm SEM of 6 rats per group;

${ }^{*} \mathrm{P}<0.05$; ${ }^{* *} \mathrm{P}<0.01$; ${ }^{* * *} \mathrm{P}<0.001$; ${ }^{* * *} \mathrm{P}<0.0001$.

\section{Discussion}

This study investigated a highly selective antagonist of CaV2.1 channels and the effects on learning and memory performance in a rat model. We found that the intrahippocampal injection of kainic acid strongly impaired the passive avoidance, spatial learning and memory in rats, as assessed by established tests. Our findings were supported by those of previous studies, showing that kainic acid's neurotoxicity results in cognitive impairment in rats $[19,29]$. It is known that kainic acid binds to specific receptors in the hippocampus and causes excitotoxicity effects $[10,19,20]$. Subsequently, the influx of excess calcium into neurons causes increased release of neurotransmitters. The overactivity of $\mathrm{CaV} 2.1$ channels causes neuronal deterioration induced by P38, generation of large amounts of free radicals, and activation of caspases, which eventually lead to apoptosis $[10,11,19$, $20,29,33,34]$. Our findings confirmed that the passive avoidance and Morris water maze tests were appropriate methods for the evaluation of learning and memory performance in rats [20, 21].

The experimental rat model that was used in this study appropriately demonstrated impairment in the behav- 
ioral functions including memory, learning and recognition. Thus, our findings suggest that the CaV2.1 channels have an essential role in the animal's behaviors [8]. Prior to the current study, there was no experimental evidence to demonstrate the effect of the over-stimulated CaV2.1 channels on the behavioral performance in animal models. An earlier study on the function of omegalycotoxin has suggested modulatory effect of this agent on CaV2.1 channels in rats' cerebellar Purkinje cells and hippocampal CA1-CA3 neurons $[25,26]$. Our findings further suggest that the release of neurotransmitters in the synaptic cleft can be modified by omega-lycotoxin. Further, the excitotoxicity effect caused by kainic acid can be modulated by omega-lycotoxin.

Our results demonstrated that the memory and learning impairment induced by kainic acid were improved by the administration of omega-lycotoxin. The latency in the passive avoidance test, indicative of learning, was increased in the rats receiving omega-lycotoxin compared to the group treated with kainic acid only. Likewise, the time spent in the target quadrant in a Morris water maze test, which reflects the spatial learning and memory performance, was enhanced in the animals receiving omega-lycotoxin as compared to the group treated with kainic acid only.

To date, no study has been conducted on the effect of omega-lycotoxin on learning and memory performance in animal models. Our findings provided experimental evidence that omega-lycotoxin prevented and reverted the learning and memory impairment induced by kainic acid through modulating the overactive CaV2.1 channels in the hippocampus. Our findings are consistent with those reported by previous studies on the binding of omega-lycotoxin to CaV2.1 channels [25, 26]. Our results revealed that the administration of omega-lycotoxin in higher doses $(2 \mu \mathrm{g}$ versus $0.5 \mu \mathrm{g})$ caused greater improvement in memory and learning, and reverted the neuronal deterioration caused by their exposure to kainic acid. This finding implies that $2 \mu \mathrm{g}$ might be the optimal dose of omega-lycotoxin in the rats used in this study.

Meanwhile, the post-treatment groups showed higher degrees of improvement than the pre-treatment ones, suggestive of the modulatory properties of this toxin [35]. As modulators do not affect the basal activity of neuronal channels, they show higher effects if used as a post-treatment. As a concluding remark, omega-lycotoxin may effectively protect the neurons against the destructive effects of kainic acid, improves learning and memory impairment by modulating over-stimulated CaV2.1 channels, and leads to normalizing the release of the neurotransmitters. Finally, it can control the activities of excitatory synapses, such as NMDA and kainic acid receptors.

Limitation of the study: A major limitation of this study was the high mortality rate of rats during and after the surgery, performed by Strotex method, due to injection of kainic acid.

Recommendation for future research: We recommend that future studies be conducted on the isolation of the crude venoms from the Iranian spiders followed by the venoms' fractionation of the active components, determination of the mechanisms of action involved in the excitotoxicity.

\section{Conclusions}

Omega-lycotoxin, an agent from the wolf spider, has modulating effects on calcium V2.1 channels, which enhances learning and memory in a rat model. Based on our findings, we have provided experimental evidence that the administration of omega-lycotoxin at $0.5-2 \mu \mathrm{g}$ in rats, pretreated with kainic acid, improved their memory and learning performance. This toxin is likely to induce this effect by modulating the Cav2.1 channels. Further, we demonstrated that increasing the doses of omegalycotoxin caused further improvement in the rats' memory and learning performance. In summary, the data analyses revealed that pre-treatment and post-treatment with omega-lycotoxin may lead to different levels of improvement on learning and memory deficits in a rat model. Omega-lycotoxin is likely to be a potential future pharmaceutical candidate for the management of cognitive deficits in humans.

\section{Ethical Considerations}

\section{Compliance with ethical guidelines}

All experimental procedures were approved by the Animal Ethics Committee of Isfahan University of Medical Science (Code: IR.MUI.RESEARCH. REC.1398.089). This paper was extracted from the Master's degree thesis materials of the corresponding author.

\section{Funding}

This study was funded and conducted at the Department of Pharmacology and Toxicology, School of Pharmacy and Pharmaceutical Sciences, Isfahan University of Medical Sciences, Isfahan, Iran (Grant No.: 397778). 


\section{Author's contributions}

Conceptualization and supervision: Mohammad Keimasi, Ali Hosseini-Sharifabad; Data acquisition: Mohammad keimasi; Writing the manuscript: All authors.

\section{Conflict of interest}

The authors declared no conflict of interest.

\section{Acknowledgements}

The authors acknowledge the support of the management and staff of the Department of Pharmacology and Toxicology and Pharmaceutical Sciences Research Center, School of Pharmacy and Pharmaceutical Sciences, Isfahan University of Medical Sciences.

\section{References}

[1] Olivares D, K Deshpande V, Shi Y, Lahiri DK, Greig NH, Rogers JT, et al. N-methyl D-aspartate (NMDA) receptor antagonists and memantine treatment for Alzheimer's disease, vascular dementia and Parkinson's disease. Curr Alzheimer Res. 2012; 9(6):746-58. [DOI:10.2174/156720512801322564] [PMID] [PMCID]

[2] Danysz W, Parsons CG. Alzheimer's disease, $\beta$-amyloid, glutamate, NMDA receptors and memantine-searching for the connections. Br J Pharmacol. 2012; 167(2):324-52. [DOI:10.1111/j.1476-5381.2012.02057.x] [PMID] [PMCID]

[3] Shao W, Peng D, Wang X. Genetics of Alzheimer's disease: From pathogenesis to clinical usage. J Clin Neurosci. 2017; 45:1-8. [DOI:10.1016/j.jocn.2017.06.074] [PMID]

[4] Hosseini-Sharifabad A, Rabbani M, Seyed-Yousefi Y, Safavi M. Magnesium increases the protective effect of Citicoline on Aluminum Chloride-induced cognitive impairment. Clin Psychopharmacol Neurosci. 2020; 18(2):241-8. [DOI:10.9758/ cpn.2020.18.2.241] [PMID] [PMCID]

[5] Teclise NG. Characterization of splice variations in the EFhand of the Cav 2.1 P/Q-Type voltage-gated calcium channel in the central nervous system [MSc. thesis]. Singapore: National University of Singapore; 2008. https:/ / scholarbank. nus.edu.sg/handle/10635/16013

[6] Jung D, Hwang YJ, Ryu H, Kano M, Sakimura K, Cjo J. Conditional knockout of Cav2.1 disrupts the accuracy of spatial recognition of CA1 place cells and Spatial/Contextual recognition behavior. Front Behav Neurosci. 2016; 10:214. [DOI:10.3389/fnbeh.2016.00214.] [PMID] [PMCID]

[7] Belardetti F, Zamponi GW. Calcium channels as therapeutic targets. Wiley Interdisciplinary Reviews: Wiley Interdiscip Rev Membr Transp Signal. 2012; 1(4):433-51. [DOI:10.1002/wmts.38]

[8] Mallmann RT, Elgueta C, Sleman F, Castonguay J, Wilmes $\mathrm{T}$, van den Maagdenberg A, et al. Ablation of Ca V 2.1 voltage-gated $\mathrm{Ca}_{2}{ }^{+}$channels in mouse forebrain generates mul- tiple cognitive impairments. PLoS One. 2013; 8(10):e78598. [DOI:10.1371/journal.pone.0078598] [PMID] [PMCID]

[9] Dobrek Ł, Thor P. Glutamate NMDA receptors in pathophysiology and pharmacotherapy of selected nervous system diseases. Postepy Higieny i Medycyny Doswiadczalnej. 2011; 65:338-46. [DOI:10.5604/17322693.946637] [PMID]

[10] Zhang XM, Zhu J. Kainic acid-induced neurotoxicity: Targeting glial responses and glia-derived cytokines. Curr Neuropharmacol. 2011; 9(2):388-98. [DOI:10.2174/1570159117955 96540] [PMID] [PMCID]

[11] Lai TW, Zhang S, Wang YT. Excitotoxicity and stroke: Identifying novel targets for neuroprotection. Prog Neurobiol. 2014; 115:157-88. [DOI:10.1016/j.pneurobio.2013.11.006] [PMID]

[12] Martin HG, Wang YT. Blocking the deadly effects of the NMDA receptor in stroke. Cell. 2010; 140(2):174-6 [DOI:10.1016/j.cell.2010.01.014] [PMID]

[13] Chamorro Á, Dirnagl U, Urra X, Planas AM. Neuroprotection in acute stroke: targeting excitotoxicity, oxidative and nitrosative stress, and inflammation. Lancet Neurol. 2016; 15(8):869-81. [DOI:10.1016/S1474-4422(16)00114-9]

[14] Binvignat O, Olloquequi J. Excitotoxicity as a target against neurodegenerative processes. Curr Pharm Des. 2020; 26(12):1251-62. [DOI:10.2174/1381612826666200113162641] [PMID]

[15] Szydlowska K, Tymianski M. Calcium, ischemia and excitotoxicity. Cell Calcium. 2010; 47(2):122-9. [DOI:10.1016/j. ceca.2010.01.003] [PMID]

[16] Hynd MR, Scott HL, Dodd PR. Glutamate-mediated excitotoxicity and neurodegeneration in Alzheimer's disease. Neurochem Int. 2004; 45(5):583-95. [DOI:10.1016/j.neuint.2004.03.007] [PMID]

[17] Vincent P, Mulle C. Kainate receptors in epilepsy and excitotoxicity. Neuroscience. 2009; 158(1):309-23. [DOI:10.1016/j. neuroscience.2008.02.066] [PMID]

[18] Amini E, Golpich M, Farjam AS, Kamalidehghan B, Mohamad Z, Ibrahim NM, et al. Brain lipopolysaccharide preconditioning-induced gene reprogramming mediates a tolerance state in electroconvulsive shock model of epilepsy. Front Pharmacol. 2018; 9:416. [DOI:10.3389/fphar.2018.00416] [PMID] [PMCID]

[19] Khodamoradi M, Asadi-Shekaari M, Esmaeili-Mahani S, Esmaeilpour K, Sheibani V. Effects of genistein on cognitive dysfunction and hippocampal synaptic plasticity impairment in an ovariectomized rat kainic acid model of seizure. Eur J Pharmacol. 2016; 786:1-9. [DOI:10.1016/j.ejphar.2016.05.028] [PMID]

[20] Jia C, Han S, Wei L, Dang X, Niu Q, Chen M, et al. Protective effect of compound Danshen (Salvia miltiorrhiza) dripping pills alone and in combination with carbamazepine on kainic acid-induced temporal lobe epilepsy and cognitive impairment in rats. Pharm Biol. 2018; 56(1):217-24. [DOI:10.1080 /13880209.2018.1432665] [PMID] [PMCID]

[21] Wang CC, Ho YH, Hung CF, Kuo JR, Wang SJ. Xanthohumol, an active constituent from hope, affords protection against kainic acid-induced excitotoxicity in rats. Neurochem Int. 2020; 133:104629. [DOI:10.1016/j.neuint.2019.104629] [PMID]

[22] Zhang F-X, Sun QJ, Zheng XY, Lin YT, Shanh W, Wang $\mathrm{AH}$, et al. Abnormal expression of synaptophysin, SNAP-25, 
and synaptotagmin 1 in the hippocampus of kainic acid-exposed rats with behavioral deficits. Cell Mol Neurobiol. 2014; 34(6):813-24. [DOI:10.1007/s10571-014-0068-3] [PMID]

[23] Creeley C, Wozniak DF, Labruyere J, Taylor GT, Olney JW. Low doses of memantine disrupt memory in adult rats. J Neurosci. 2006; 26(15):3923-32. [DOI:10.1523/JNEUROSCI.4883-05.2006] [PMID] [PMCID]

[24] Sun X, Zhong J, Wang D, Xu J, Su H, An C, et al. Increasing glutamate promotes ischemia-reperfusion-induced ventricular arrhythmias in rats in vivo. Pharmacology. 2014; 93(1-2):4-9. [DOI:10.1159/000356311] [PMID]

[25] Pluzhnikov K, Vassilevski A, Korolkova Y, Fisyunov A, Iegorova $\mathrm{O}$, Krishtal $\mathrm{O}$, et al. $\omega$-Lsp-IA, a novel modulator of P-type Ca2 ${ }^{+}$channels. Toxicon. 2007; 50(7):993-1004. [DOI:10.1016/j.toxicon.2007.07.004] [PMID]

[26] Fisyunov A, Pluzhnikov K, Molyavka A, Grishin E, Lozovaya N, Krishtal O. Novel spider toxin slows down the activation kinetics of P-type $\mathrm{Ca}^{+}$channels in Purkinje neurons of rat. Toxicology. 2005; 207(1):129-36. [DOI:10.1016/j. tox.2004.09.005] [PMID]

[27] Kajani AA, Mofid MR, Abolfazli K, Hosseini Tafreshi SA. Encapsulated activated charcoal as a potent agent for improving taxane synthesis and recovery from cultures. Biotechnol Appl Biochem. 2010; 56(2):71-6. [DOI:10.1042/BA20090344] [PMID]

[28] Kajani AA, Moghim S, Mofid MR. Optimization of the basal medium for improving production and secretion of taxanes from suspension cell culture of Taxus baccata L. Daru. 2012; 20(1):54. [DOI:10.1186/2008-2231-20-54] [PMID] [PMCID]

[29] Miltiadous P, Stamatakis A, Stylianopoulou F. Neuroprotective effects of IGF-I following kainic acid-induced hippocampal degeneration in the rat. Cell Mol Neurobiol. 2010; 30(3):347-60. [DOI:10.1007/s10571-009-9457-4] [PMID]

[30] Paxinos G, Watson C. The rat brain in stereotaxic coordinates. London; Elsevier/Academic: 2009. https://books. google.com/books?id=sQNfmwEACAAJ\&dq

[31] Safavi M, Hosseini-Sharifabad A, Seyed-Yousefi Y, Rabbani M. Protective effects of citicoline and benfotiamine each alone and in combination on streptozotocin-induced memory impairment in mice. Clin Psychopharmacol Neurosci. 2020; 18(1):81-92. [DOI:10.9758/cpn.2020.18.1.81] [PMID] [PMCID]

[32] Akbari E, Naghdi N, Motamedi F. Functional inactivation of orexin 1 receptors in CA1 region impairs acquisition, consolidation and retrieval in Morris water maze task. Behav Brain Res. 2006; 173(1):47-52. [DOI:10.1016/j.bbr.2006.05.028] [PMID]

[33] Amini E, Rezaei M, Ibrahim NM, Golpich M, Ghasemi R, Mohamad Z, et al. A molecular approach to epilepsy management: from current therapeutic methods to preconditioning efforts. Mol Neurobiol. 2015; 52(1):492-513. [DOI:10.1007/ s12035-014-8876-5] [PMID]

[34] Rothan HA, Amini E, Faraj FL, Golpich M, Teoh TC, Ghola$\mathrm{mi}$ K, et al. NMDA receptor antagonism with novel indolyl, 2-(1, 1-Dimethyl-1, 3-dihydro-benzo [e] indol-2-ylidene)malonaldehyde, reduces seizures duration in a rat model of epilepsy. Scientific Reports. 2017; 7(1):1-10. [DOI:10.1038/ srep45540] [PMID] [PMCID]
[35] Nimmrich V, Gross G. P/Q-type calcium channel modulators. Br J Pharmacol. 2012; 167(4):741-59. [DOI:10.1111/j.14765381.2012.02069.x] [PMID] [PMCID] 